\title{
The Structure of Group Therapy for Anxiety in Children and Adolescents with Autism Spectrum Disorder in a CAMHS Setting in the UK
}

\section{Abstract}

This article is a review of Group Therapy for anxiety in children and adolescents with high functioning Autism Spectrum Disorder (ASD). It first provides a literature review of available papers to outline some of the key features of ASD, its comorbidity with anxiety and the emergence of effective intervention strategies for use in this group of individuals. It also looks at adaptations that were commonly suggested by these studies to tailor CBT and counselling interventions to the specific needs of ASD population. Then, it describes the structure of a group therapy treatment in a CAMHS setting in the UK.

Keywords: Autism; Group therapy; CBT; MASSI

Received: January 26, 2018; Accepted: February 06, 2018; Published: February 20, 2018

\section{Description of the Disorder}

Autism Spectrum Disorders term used to refer to the set of pervasive developmental disorders including Autism, Asperger's Syndrome and Pervasive Developmental Disorder Not Otherwise Specified. This group of disorders was characterised by pervasive difficulties in the key areas of social communication interactions and restrictive repetitive behaviours or activities [1,2]. The DSMIV used the criteria of language delay and intellectual disability to diagnostically differentiate Autism from Asperger Syndrome.

In the most recent version of the Diagnostic and Statistical Manual of Mental Disorders (the DSM-V) [1], released by The American Psychiatric Association in May 2013, these familiar autism spectrum disorders were officially eliminated and now incorporated all into the single diagnosis of autism spectrum disorder. The Work Group believed a single umbrella disorder would improve the diagnosis of ASD without limiting the sensitivity of the criteria, or substantially altering the figure of children being diagnosed and would also allow clinicians defining a more beneficial treatment plan for patients by defining an accurate diagnosis as a first step.

The epidemiology of ASD approximately estimated the prevalence from 30 to 60 in 10,000, although increasing awareness of the disorder has led to greater rates of diagnosis [3]. According to the latest estimates by the United States Centre for Disease Control, ASD may even be as common as 1 in every 68 children [4] (Table 1).

\section{Hadi Shaker Naeeni ${ }^{1 *}$ and Uttom Chowdhury ${ }^{2}$}

\author{
1 Hertfordshire Partnership University NHS \\ Foundation Trust, CAMHS North, 2nd \\ Floor, Saffron Ground, Ditchmore Lane, \\ Stevenage SG1 3LJ, UK \\ 2 East London NHS Foundation Trust, UK
}

\begin{abstract}
*Corresponding author:
Dr Hadi Shaker Naeeni

\section{hadi.shaker@doctors.org.uk}

Consultant Psychiatrist, Hertfordshire Partnership University NHS Foundation Trust, CAMHS North, 2nd Floor, Saffron Ground, Ditchmore Lane, Stevenage SG1 3LJ, UK.

Citation: Shakernaeeni H, Chowdhury U. The Structure of Group Therapy for Anxiety in Children and Adolescents with Autism Spectrum Disorder in a CAMHS Setting in the UK. J Child Dev Disord. 2018, 4:1.

Difficulties with a clear-cut estimate of ASD prevalence related possibly to early studies relying on surveys of clinically identified rather than community-based cases, which may have resulted in cases not in treatment being missed and possibly only the most severe cases being recorded. Previous estimates may also have been different due to previous case definitions. As both sampling methods have improved and also different diagnostic instruments are used, more cases have been identified and there has been a better delineation of ASD from other psychiatric disorders. As

Table 1 Identified prevalence of autism spectrum disorder ADDM Network 2000-2012 combining data from all sites.

\begin{tabular}{|c|c|c|c|c|}
\hline $\begin{array}{c}\text { Surveillance } \\
\text { year }\end{array}$ & $\begin{array}{c}\text { Birth } \\
\text { year }\end{array}$ & $\begin{array}{c}\text { Number of } \\
\text { ADDAM Sites } \\
\text { reporting }\end{array}$ & $\begin{array}{c}\text { Prevalence per } \\
1,000 \text { Children } \\
\text { (Range) }\end{array}$ & $\begin{array}{c}\text { This is } \\
\text { about } 1 \text { in } \mathrm{X} \\
\text { children }\end{array}$ \\
\hline 2000 & 1992 & 6 & $6.7(4.5-9.9)$ & 1 in 150 \\
\hline 2002 & 1994 & 14 & $6.6(3.3-10.6)$ & 1 in 150 \\
\hline 2004 & 1996 & 8 & $8.0(4.6-9.8)$ & 1 in 125 \\
\hline 2006 & 1998 & 11 & $9.0(4.2-12.1)$ & 1 in 110 \\
\hline 2008 & 2000 & 14 & $11.3(4.8-21.2)$ & 1 in 88 \\
\hline 2010 & 2002 & 11 & $14.7(5.7-21.9)$ & 1 in 68 \\
\hline 2012 & 2004 & 11 & $14.6(8.2-24.6)$ & 1 in 68 \\
\hline
\end{tabular}


more children with ASD are identified, there will be a likely rise in the number of families and children seeking treatment.

\section{ASD Comorbidity with Anxiety}

There are very high levels of co-morbid psychiatric difficulties among this population with estimates ranging of $7 \%$ to $15 \%$. Anxiety related concerns are among the most common presenting problems for school age children and adolescents with ASD [5].

Several studies have examined the prevalence of anxiety in young people with ASD. A review by White et al. [6] of 11 such studies reported prevalence of a broader range of $13.6 \%$ to $84 \%$. This large range in the prevalence may be accounted for by the difference in the methods used to measure anxiety, definitions and in diagnostic subtypes. Some of the most frequently reported anxiety disorders and symptoms seen in children with ASD were simple phobia, generalised anxiety disorder, separation anxiety disorder, obsessive-compulsive disorder and social phobia.

More recently, a systematic review of the literature by Van Steensel et al. identified 31 studies involving 2,121 young people with ASD aged younger than 18 years and where the presence of anxiety disorder was assessed using standardized questionnaires or diagnostic interviews. Across studies, $39.6 \%$ of young people with ASD had at least one comorbid DSM-IV anxiety disorder, the most frequent being specific phobia (29.8\%) followed by OCD $(17.4 \%)$ and social anxiety disorder (16.6\%). Associations were found between the specific anxiety disorders and ASD subtype, age, IQ and assessment method (questionnaire versus interview) [7].

Studies have also looked at prevalence rates of anxiety among ASD individuals compared to other populations, for instance compared to groups of typically developing children, those with ASD had a higher occurrence of anxiety $[8,9]$. Children with ASD were also more likely to be diagnosed with an anxiety disorder and/or have more intense anxiety symptoms in comparison with other at risk groups, i.e. children with conduct disorders and learning disabilities $[10,11]$.

Formal diagnosis of anxiety disorders in this group is hard for therapists due to overlap between the comorbid anxiety and core symptoms of ASD. Several anxiety disorders in DSM- IV and ICD 10 had autism as an exclusion criterion, implying that an anxious processing style is a feature of ASD.

The development of anxiety among children with ASD may relate to their cognitive impairment as they may lack the cognitive flexibility to generate strategies to adapt to varying circumstances and may experience distress over even trivial changes in the environment. The information processing style in children with ASD termed 'weak central coherence' is similar to non ASD anxious children whereby they selectively attend to threatening cues which results in the misinterpretation of ambiguous situations as threatening $[12,13]$. As a consequence, these cognitive deficits can result in a repertoire of social and communication difficulties resulting in children experiencing severe difficulties in social relationships which may in turn lead to the development of anxiety [14].
If a child or adolescent has a co-occurring anxiety disorder, this could further negatively impact on the overall social impairment associated with ASD. This could impact on the child's or adolescent's ability to take part in activities at school, home, or within the community. Children with significant anxiety symptoms are at risk for serious educational difficulties, later unemployment, substance abuse and other psychiatric problems [15].

\section{Introduction: Group Therapy Treatment for children}

Group therapy is defined as a model of therapy, in which two or more patients meet with one or more therapists to alter their behavioural and cognitive patterns [16]. Moreno, as the father of group psychotherapy, represented the first attempts to treat people in groups rather than exclusively in one-to-one settings (Moreno 1953, 1964). Since then, the model of group therapy has evolved through different stages.

Group therapy also presented a more cost-effective method of treating the large number of children and adolescents with anxiety disorders referred to Child and Adolescent Mental Health Services (CAMHS). It was shown to help children with their socio-emotional and peer relationships difficulties, social cues understanding and processing problems and planning appropriate response to these cues [17].

The classic model of group therapy with adults would not be helpful nor practical with children and needed to be modified to accommodate the special needs of children and in particular children with ASD. Some of more common adaptations are described here.

The long-established psychoanalytic approach was practised in the field of group therapy for children and adolescents for many years. However, Yalom pioneered a new process of group therapy and introduced a structured planning based on the cognitivebehavioural group model and guidelines. For example, planning fun, attractive activities, initiating and maintaining balanced participation and increasing disclosure among members were related to the process of the group. Guidelines such as always planning sessions, determining relevant themes for group based on membership and targeted group goal and providing validation assignments were related to the cognitive-behavioural approach [18-22].

Drumm reported group intervention effective with positive outcomes in primary goals, e.g. social skill, problem solving and conflict resolution in a particular group, which could then also result in further secondary changes e.g. increased feelings of belonging, normalizing of one's experiences and increasing peer esteem and self-esteem.

A number of quasi-experimental and qualitative accounts confirmed that activity group therapy with neurotypically developed children and adolescents could help to reduce anxiety, aggression and delinquent behaviours and increase performance at school and engagement in groups and help them to feel in control of their lives [22]. 
'Leading the Way' [22] was a remarkable example of an activity group therapy showing how modifying group therapy could meet the need of children who lived with HIV. This was an approach derived from play therapy that included a mix of guided conversations, socio-recreation activities such as games, art and music that allowed direct and indirect self-expression to deliver guided discussions, psycho-education and psychotherapy to explore difficult issues and feelings about HIV/AIDS and encourage risk-reduction behaviours.

As another example, Eichstedt et al. used The Worry Warriors Program, a manualized CBT group program adapted from the Taming Worry Dragons manual developed by Garland and Clark $[23,24]$. It was appropriate for neurotypically developed children age 8-12 year and involved 12 weekly sessions of 90-minutes duration each with six therapists conducting in pairs.

Tobon et al. in combination with Chu and Kendall's study, looked at a different aspect which was the patterns of children's involvement over time in group cognitive behavioural treatment (GCBT) program for anxiety versus individual therapy and recommended using an adapted version of the Child Involvement Rating Scale (CIRS) in group therapy, similar to the measure of involvement used in individual therapy.

Adolescents with ASD often desire friendships despite their social skills difficulties and express concerns about their lack of reciprocal friendships and difficulties in maintaining and sustaining friendships. Unsurprisingly, poor social skills in ASD have been linked to significant levels of anxiety, including compulsive and ritualistic behaviour, irrational fears and beliefs, depression, increased aggression and poor relationships with parents and teachers [25].

Klinger and Williams recommended a "compensation" approach that many successful social skill interventions use, in which children with ASD were explicitly taught how to understand and interpret social cues and behaviours to compensate for their lack of implicit understanding of social information. Many empirically supported social skills interventions utilized similar techniques including incidental teaching, social stories and scripts, roleplays, self-monitoring and peer-mediated activities, e.g. peer education and peer buddy. For example, Lopata and colleagues derived their social skills curriculum for high-functioning school-age children with ASD from the Skill-streaming program, which followed a specific stepwise procedure for teaching, modelling, role-playing and feedback on social skills [25].

Mundy et al. found the structured and linear format of group therapy interfering with the process of matching intervention techniques to patients' characteristics. Therefore, they considered the heterogeneity of presenting phenotypes in highfunctioning ASD and presumably corresponding variations in underlying pathology in order to tailor intervention techniques to a patient's specific characteristics and strengths $[26,27]$.

Liber et al. [28] proposed that providing anxious children with treatment in a CBT group format could conceptually reinforce their relationships with peers, give those peers modelling, normalise anxiety and expose them to social situations which were more representative of daily life experiences.

\section{Literature Review of Group Therapy in Treatment of Children with ASD and Anxiety}

Several case studies and exploratory clinical trials have suggested that group-therapy CBT intervention may help lessen anxiety symptoms in children with ASD [29-32] (Table 2).

Chalfant et al. reported superior anxiety outcomes of a 16week group therapy CBT intervention for children with ASD and concurrent anxiety disorders who were in the immediate treatment group when compared to the waitlist group. Limitations of this study were that the therapists rather than independent evaluators blind to treatment assignment administered the posttreatment diagnostic interviews and that treatment fidelity was not assessed. Thus, whilst CBT might have been a promising intervention modality for the ASD population, methodological characteristics of the extant studies precluded conclusions about efficacy.

Sofronoff et al. [31] evaluated two variants of a 6-week CBT program in a group-therapy format that focused on emotion recognition and cognitive restructuring for children with Asperger syndrome. Participating children were not diagnosed with anxiety disorders at pre-treatment stage per se. However, parent-report measures showed declines in child anxiety symptoms in the CBT groups compared to a waitlist group.

Wood et al. [26] used a fully modular individually-focused CBT intervention developed for their study to promote optimal treatment outcomes, as one of the first of its kind to be empirically evaluated in the ASD population. They also used fear hierarchies that included steps toward making friends.

Reaven et al. [33] studied thirty-three children with highfunctioning ASD and their parents who participated in a manualized cognitive behavioural group treatment aimed at reducing the severity of anxiety symptoms. Parent-child dyads entered into either an active treatment condition or wait list control condition. Results indicated significant reductions in parents' reports of anxiety symptoms after the delivery of the group treatment, compared with the wait list control condition. The findings of this study were promising, especially in light of the high rates of comorbidity between ASD and anxiety.

Later on, Reaven et al. randomized fifty children with highfunctioning ASD and anxiety to group CBT or treatment-as-usual (TAU) for 12 weeks; initial results suggested that a group CBT intervention specifically developed for children with ASD may be effective in decreasing anxiety. Limitations of this study included small sample size, lack of an attention control group and use of outcome measures normed with typically developing children.

\section{Multimodal Anxiety and Social Skills Intervention (MASSI)}

White et al. [34] used Multimodal Anxiety and Social Skills Intervention (MASSI) and included group therapy for social skills training among their treatment modalities to offer the 
Table 2 Published clinical trials of group therapy.

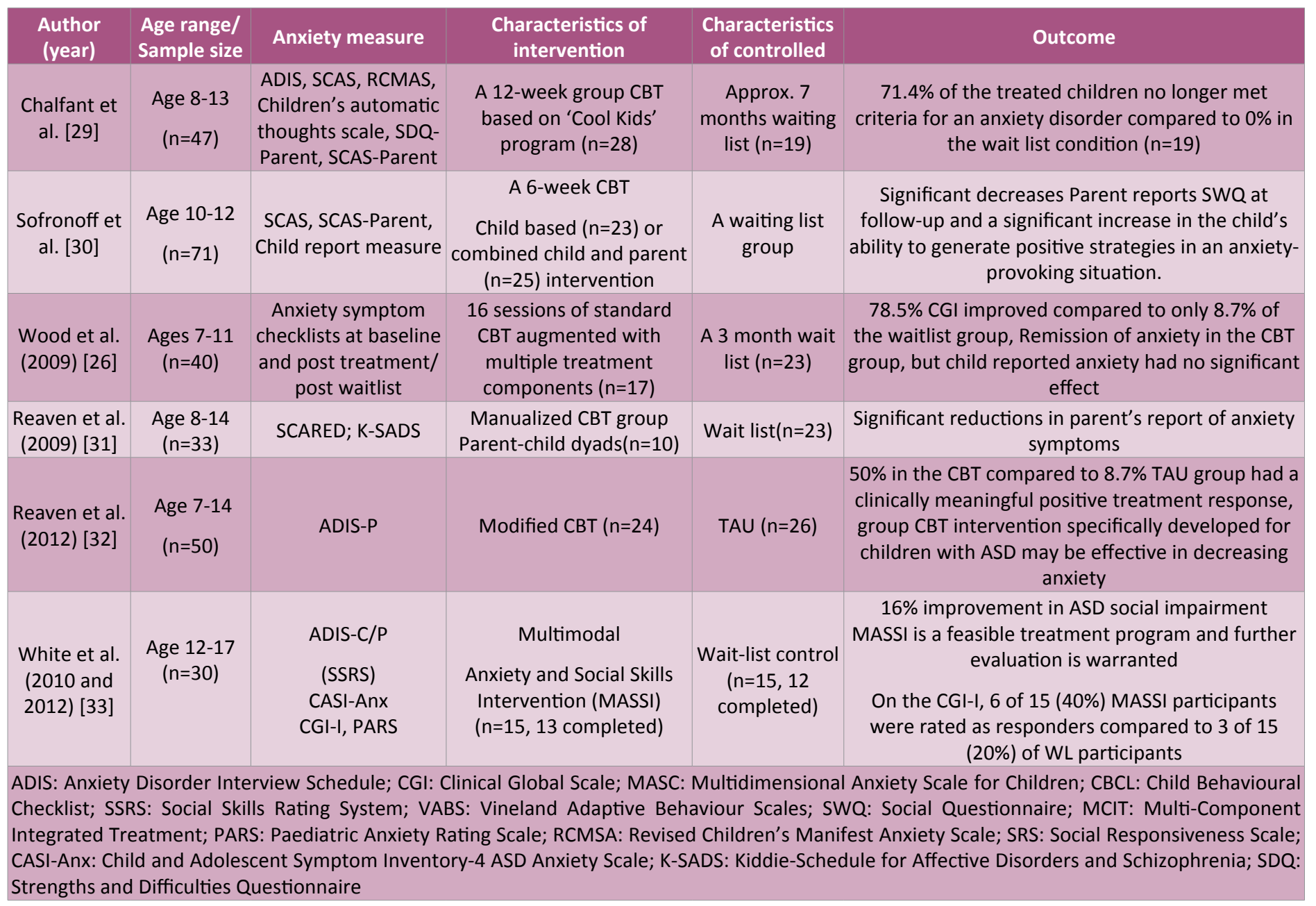

opportunity to practice social skills in a relatively naturalistic yet highly supportive environment. The therapy was designed as a CBT program and applied behaviour analysis that targeted concurrent anxiety and social disability in adolescents with ASD via individual therapy, group social skills training and parent coaching. MASSI was developed specifically for adolescents with high functioning autism spectrum disorders (HFASD) accompanied by moderate to severe anxiety problems. The 'dual focus' in treatment was based on the notion that unaddressed anxiety problems could undermine the benefits that might otherwise be realized with a 'pure' social skills intervention. Adolescents with high levels of anxiety might be less able to make use of the social skills training, avoid opportunities to practice newly learned social skills, or both.

The decision of making an integrated intervention, i.e. group sessions delivered along with individual sessions was based on the assumption that combined treatment would protect against attrition and promote more rapid improvement than sequential treatment. The group treatment modality began approximately 3 weeks after the beginning of individual therapy and continued concurrently throughout the rest of the individual therapy.

All participants in MASSI were invited to attend all the group sessions. The group meetings each lasted 75 minutes. Each participant had his or her own identified clinician for individual therapy sessions which also promoted social skills development; this therapist also co-led the participant's group sessions. Thus, for each cohort of three MASSI participants, there were two therapists. In each group of three subjects with an ASD, there was one unaffected peer tutor (volunteer of similar age from the community), who offered age-appropriate modelling and feedback to the MASSI participants. Video-recorded sessions for reliability were selected to ensure the therapists' ratings of fidelity.

The group component emphasized behavioural practice within a supportive environment to minimise the information processing weaknesses associated with ASD. The group component was meant to offer a positive social learning experience with peers, which can further motivate adolescents to continue to evolve their skills and to act on making acquaintances. A positive group experience could also reduce the inclination to escape social situations.

The content of the seven group sessions was the same for all participants, as the skills covered, e.g. how to join into peer groups, had broad applicability for adolescents with ASD (Table 3). 
Table 3 Social skills building modules and seven group sessions.

\begin{tabular}{|c|}
\hline White 2010 social skills building modules \\
\hline $\begin{array}{c}\text { Initiating with peers } \\
\text { Conversational skills } \\
\text { Flexibility and recognizing the cues of others } \\
\text { Handling rejection }\end{array}$ \\
\hline Group practice modules \\
\hline $\begin{array}{c}\text { Introduction } \\
\text { Talking to peers } \\
\text { Following a conversation } \\
\text { Emotion regulation } 1 \text { [Recognizing Cues] } \\
\text { Emotion regulation } 2 \text { [Handling It] } \\
\text { Entering a group } \\
\text { Social skills } 101\end{array}$ \\
\hline
\end{tabular}

\section{Method}

This group therapy treatment was part of a randomized casecontrol study, which compared Cognitive Behavioural Therapy, i.e. the MASSI vs. Counselling for Adolescents with Autistic Spectrum Disorder who had co-existing Anxiety. The study obtained ethics approval from East of England Ethics Committee. We used MASSI as devised by White et al.

20 out of 43 adolescents aged from 12 to 16, with diagnosis of ASD (confirmed by ADOS [34] and AID-R [35]) and anxiety symptoms (by ADIS-C/P [36]), who were under the care of Children and Adolescent Mental Health Service, attended the group therapy. The participants included 12 males and 8 females.

Our group therapy took place in an outpatient CAMHS specialist clinic at Bedfordshire. The available room was a multi-functional family therapy room with some distractions, such as recording equipment like camera and a one-way mirror, spare tables and chairs, painting materials and books. We were aware that the place and room setting might have an effect on participation of group members. We were however able to have the same room throughout the treatment which helped with consistency.

In CBT groups we focused on main topics including introduction and talking to peers, following a conversation, emotion regulation, entering a group and social skills.

In counselling groups, we focused on main themes which participants contributed to, including talking about feelings, developing friendships and communication skills.

We used small group discussions, role play exercises and feedbacks on the spot. During and after each group session we took notes about interactions and dynamics.

Our group treatment modality began about 3 weeks after the beginning of individual therapy and continued concurrently throughout the rest of the individual therapy. We used a time bound closed group using a structured approach for each individual. The therapy included five 45-minute group therapy sessions, occurring approximately every other week and each session followed by a ten-minute check in with parents. Sessions' time was after school and followed the school calendar to minimise interference with school attendance. In total, 20 participants participated in thirty sessions of group therapy to over six months.

\section{Results and Discussion}

\section{Approach and model of treatment}

Some preparation was required for the transition process of moving from one to one individual treatment to group model. We found the following points useful to think about how this process could be facilitated to allow Group Therapy participants to get well prepared;

- Pre-group sessions: one to one session with the therapist to discuss how the group works, formal/informal setting, ground rules and boundaries.

- Also exploring previous exposure to any group and experience of group activities in settings outside clinic could help them to reflect on similarities and differences of different groups.

The introduction component of the group therapy was the most sensitive phase of MASSI protocol. Handling it carefully appeared helpful for the group in order to be more comfortable and less anxious about practical points of how we wanted to deliver the group such as 'what would happen next', knowing each other, the exact length of each session and whether the process of the group discussions was scripted or spontaneous.

Each group began their first session with therapists welcoming the group and explaining their roles and who they were, frequency of sessions and also setting the ground rules and clarifying the confidentiality. We noticed most of the participants liked an ice breaking time to manage their anxiety.

The therapists began to introduce themselves, which provided the group with a model in action to help the participants to become familiar with basic social cues. Most of the participants talked about their hobbies and interests to introduce themselves to others. On one hand, there were some participants who could not introduce themselves saying 'I don't know what to say about myself'. On the other hand, there were some who wanted to tell others everything about their hobbies during introductions.

Throughout the counselling sessions, participants were encouraged to bring up different topics so that they could engage in a conversation. These included their hobbies, feelings, interests as well as their special interests such as sport, music, video games and animals. We noticed that incorporating special interests into activities significantly increased social interest and participation [37].

Participants also talked about their social difficulties. The common themes included school related issues, being bullied, dealing with rumours and difficulties with other peers and some teachers. We tried to validate their emotional experiences and feedback directly on their experiences. We focused on social skills training such as modelling reactions to bully and best ways of seeking help, how to join a group, maintain a conversation and how to use appropriate social greeting to improve their social skills and reduce the chance of more of such experiences [33].

Participants were curious and reflective about the meaning of 'being normal/not normal' and were curious to know how 
clinicians could recognise if someone had Asperger's. Participants also talked about their previous experiences of social interactions such as being part of a group, having a choice of picking up their group and being part of it, or being excluded from it. One of the participants used the term 'social lubricant' referring to a sense of humour in social situations to facilitate communication. As other examples, they could also consider pretending and sharing jokes. Similarly, in another group they found 'silence always awkward' and agreed 'talking' could reduce discomfort. On the other hand, there were a few participants who mentioned they found social interaction 'waste of time' and preferred their own company.

\section{Gender}

The most recent prevalence study in the US (CDC 2012) [4] estimated that the ratio of boys to girls with an ASD is 5:1. The strategies used in CBT are usually gender-neutral, but there may be situations, such as being bullied by girls rather than boys, which require gender-specific strategies. Clinical experience indicated that there could be advantages in having groups specifically for girls, if this was at all possible. Therefore, clinicians need to bear in mind that should a girl with ASD be the only female in the group, she might feel uncomfortable and self-conscious.

On the other hand, male/female mixed groups could well mirror the social world, e.g. school social setting, in which group members need to deal with each other mainly about sensitive emotions such as being rejected or bullied.

Our participants' male/female ratio was different from CDC 2012 because we had about 40\% girls (5:2). From a gender point of view and in contrary to boys, girls who attended the group found being around the boys rather comfortable. However, boys usually intended to pair with each other, possibly because of either sharing similar special interests such as video games/sport or being anxious about interacting with girls.

Female participants were more reflective about their selfconcept. For example, one girl in the group connected her special interests about history with her identity and self-concept when reflected on herself as 'finding myself similar to old-time people'.

Therefore, the decision about having a mixed group could depend on the peer group, members' experience and the content of their discussions.

\section{Size}

One participant reflected on the word 'group' and said that when he was invited to the group, he imagined himself 'being among fifty people and had to stand in front of a microphone to talk to others'. His imagination scared him and he was about to refuse to come, but later on he understood the setting was smaller and friendlier. This invited the thought about the usage of an essentially clearer language in future invitation letters to explain the size of the group.

Another participant's first reaction to the idea of coming to a group was that they would attend the group 'only if there was no other participant in the group'. However, their attitude shifted towards a successful group attending.
The ideal group size also matters. Our groups consisted of minimum 3 to maximum 5 adolescents, both male and female. The ideal size might be a group of 4 participants, ( 2 boys $/ 2$ girls), with two therapists.

\section{Participation}

Screening is needed to allow participant selection. Participants' factors such as their personality, special interests, sensory issues, snack choice, the severity of anxiety, level of social impairment, could not only have a massive impact on participants' engagement and non-engagement, group dynamic and pace, but also on how threatening or comforting they would perceive a group.

Atwood and Scarpa [38] recommend the group participants to be carefully selected to increase cohesion of the group. Children and adolescents with ASD are at risk of additional diagnoses or problematic behaviours, including externalising behaviours, such as impulsivity, hyperactivity and oppositionality, which can have an impact on cohesion. They also talked about the following points:

- Leader to participant ratio to allow effective monitoring and facilitation of group interactions and attention, depending on small/large groups, hyperactivity, behavioural difficulties.

- The personality of each participant and their emotional and intellectual maturity to maximise group cooperation, mutual support and the possibility of the development of friendship within or after the group sessions.

By reviewing the dynamic of the interactions of our participants, three patterns emerged from their social interactions in a group; these patterns could interchange with each other within a session or dominate a whole session but not the other. In the following diagrams, black circles are therapists, white circles are participants and arrows show the direction of interactions.

a) Slow respondent; group might become very therapist led and rely on therapists to provide the idea. There is no interaction between participants and all conversations are channelled to the therapists who draw connections between members. The group could easily become non-productive (Figure 1).

b) Appropriate respondent; interacting according to the context of the session, participants joining the conversation. There are appropriate reciprocal and spontaneous conversations (Figure 2).

c) Rapid respondent; by being over talkative or entertaining; group might become easily distracted from the goals; one participant might take over the lead and force others to listen to his or her special interest (Figure 3).

\section{Feedback}

We asked the participants about their experience of participating in the group when they finished the therapy. What emerged from the feedbacks was similar to a few of what Yalom identified as 11 characteristics that make group therapy effective; Universality, Group Cohesiveness, Altruism, Instillation of Hope, Imparting Information, Interpersonal Learning, Development of Socializing Techniques, Imitative Behaviour, Corrective Recapitulation of the Primary Family Group, Catharsis and Existential Factors. 

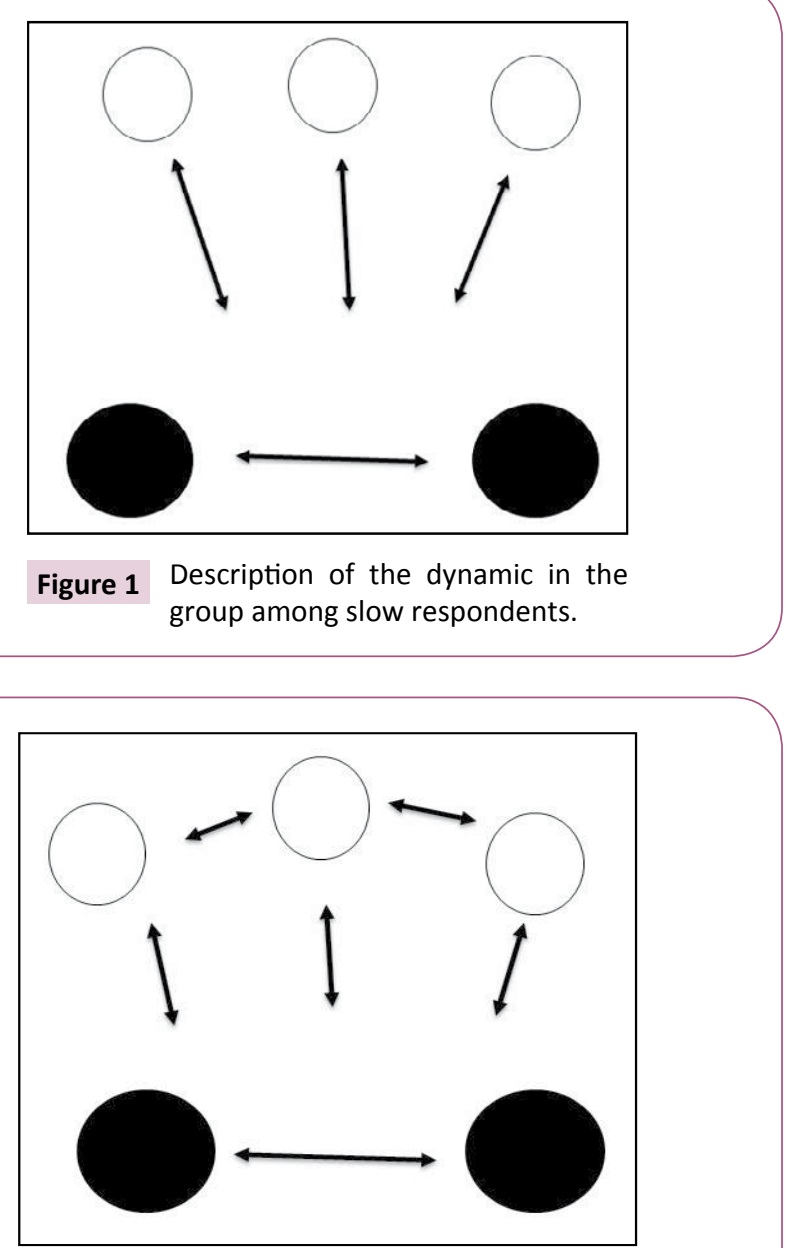

Figure 2 Description of the dynamic in the group among appropriate respondents.

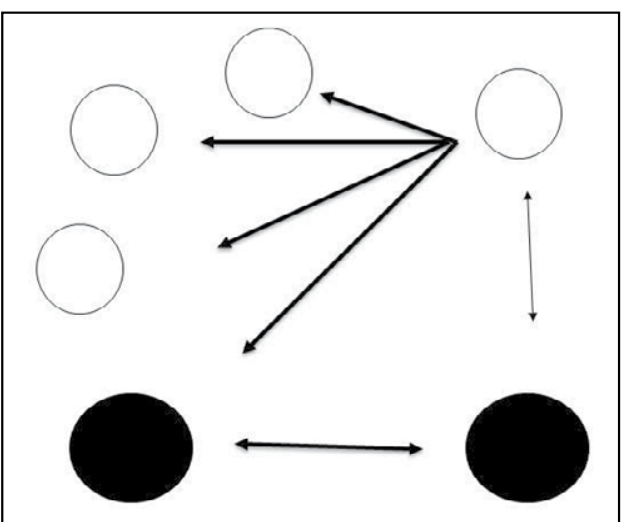

Figure 3 Description of the dynamic in the group among rapid respondents.

The foremost feedback was about how meeting other people with similar problems helped them. Universality, i.e. I'm not alone nor have unique problems, as well as the instillation of hope were the most appreciated features.

- One participant who rated their experience 5 out of 10 (pretty helpful) said that 'There seem to be a load of people with the same issue'.

Participants also appreciated peer support helpful in improving their understanding about ASD-related problems.

- One participant who rated their experience 8 out of 10 said 'Other people can tell the same way as me, I learned about different people's problems and how they tell'.

- One participant who rated their experience 5 out of 10 (pretty helpful) said that 'I learnt things about myself, ASD and Anxiety'.

We also noticed how they learned through Imparting Information (educational), Interpersonal Learning, Development of Socializing Techniques and Imitative Behaviour;

- One participant who rated their experience 8 out of 10 (very helpful) said 'Talking to someone I don't know that well about things made me feel more confident about talking to people'.

- One participant who rated their experience 10 out of 10 (very helpful) said 'Learned how to make friends'.

- One participant who rated their experience 7 out of 10 said 'How to make decisions when I have a choice'.

We also had very different feedbacks from some of the participants. Some participants found their group experience not helpful;

- One participant who rated their experience 3 out of 10 (closer to not at all helpful) said 'I have never noticed the effect of the program honestly'.

- One participant who rated their experience 3 out of 10 said 'It made me realise more how much I would like to be tested for NOT having AS and whatever else'.

Such experiences might have been due to different factors, such as wrong selection during our assessment, attending group only because they were told to, to keep their clinician or parent happy, social anxiety causing difficulty to join, different expectation from being in a group, or previous experiences of joining a group. Therefore, clinicians could help the participants by actively seeking their view about their group experience.

Parents also gave us their feedback. They informally talked to us whilst they were waiting to collect their children. They appeared to be reassured by this experience. We did not have enough resources to offer them a parallel parent group to support them; however, we felt a parallel coffee group led by an experienced facilitator such as a parent or a CAMHS practitioner could provide an enormous support.

\section{Group therapists}

Two therapists facilitated the sessions and stayed the same to provide consistency. In all groups, facilitators considered to plan sessions and determine relevant themes for each group based on membership and targeted group goal. Each participant had his or her own clinician for individual therapy in CAMHS which also promoted social skills development.

We found having two Group therapists a practical way to model communication, which helped to improve participants' social skills by modelling appropriate and suitable responses in social 
settings such as sharing appropriate information, ways of dealing with feelings and skills set of identifying thoughts and underneath feelings.

Group therapists provided instant praise on a here and now basis' for practical behaviour of participants and constructive feedbacks to help participants to use less of avoidance and more of an acceptance.

\section{Personal reflection}

First of all, we tried being sensitively aware of our own thoughts, feelings and beliefs. This awareness made us to refrain from communicating a model as too good to be real or appearing as impossible to be like us.

We were particularly very interested in thinking about the process as what we were doing and why, rather than just following the manual. Therefore, we actively talked to each other to check whether our planning fitted to the group and what was going on in the group.

We used video recording to reflect on our practice and also constantly learn from feedback and outcome. We adopted the level of our response to our participants' paces, e.g. spending enough time when someone needed to receive an empathic response appropriate to the context such as being bullied or rejected. As one of the main learning points, we could receive new perspectives by asking the participants to let us know their feedbacks.

In a 'co-therapy' relationship, one of us was a more skilled group therapist, which made the experience of being co-therapist very secure feeling for the other one. It worked well as we could plan the group together when someone was on annual leave.

\section{Conclusion}

Group therapy for children with ASD and anxiety is supported by the literature. This article explained about a CAMH service that provided a group therapy. The limitation is difficult to draw firm conclusions as numbers are low, but there are potential for further studies.

\section{References}

1 American Psychiatric Association (2013) Diagnostic and statistical manual of mental disorders. $5^{\text {th }}$ edn. text revision. Washington, DC: American Psychiatric Association.

2 Sturmey P, Fitzer A (2007) Autism spectrum disorders: Applied behaviour analysis, evidence and practice. Austin, TX: Pro-Ed; p: 289.

3 Fombonne E (2003) The prevalence of autism. JAMA 289: 87-89.

4 Christensen DL, Baio J, Braun KV, Bilder D, Charles J, et al. (2016) Prevalence and characteristics of autism spectrum disorder among children aged 8 years. Autism and Developmental Disabilities Monitoring Network. 11 Sites, United States, 2012. MMWR Surveill Summ 65: 1-23.

5 Ghaziuddin M (2002) Asperger Syndrome: Associated psychiatric and medical conditions. Focus Autism Other Dev Disabl 17: 138-144.
We used a time bound closed group using a structured approach to cognitive-behavioural and social aspect of treatment. This was an interesting process, which was appreciated by the participants and their families. They requested to receive such groups again in the future.

The therapists also learned about different aspects of group settings such as group dynamics, group composition and structure, the role of group leader and the change process in groups. It was a joyful experience to participate in young people's groups. We learned from the whole process and about their concerns and worries as well as their favourite books, readings, movies, video games and music.

Group therapy could provide a social impression similar to the real life scenario experiences and facilitate social skills learning and interpersonal communications. It also helps members learn about how others view them and what impact their behaviour could have on others.

The very different feedbacks for the different participants made it clear that despite all steps taken to match the participants and their suitability, active and on-going feedback seeking is essential to improve their participation and increase the quality of their group exercise.

If we would be able to run such groups again in future, we would try using our experience to build a group by considering better participants' selection. We would also try to have a parallel parent's support group.

\section{Acknowledgements}

We would like to thank the young people and their families who participated in the groups and helped us to understand their views and feelings.

\section{Funding Statement or Declaration of Conflicting Interests}

The authors received no financial support for the research, authorship, and/or publication of this article.

6 White SW, Oswal D, Ollendick T, Scahill L (2009) Anxiety in children and adolescents with autism spectrum disorders. Clin Psychol Rev 29: 216-229.

7 Van Steensel F, Bogels SM, Perrin S (2011) Anxiety disorders in children and adolescents with autistic spectrum disorders: A metaanalysis. Clin Child Fam Psychol Rev 14: 302-317.

8 Gillot A, Furniss F, Walter A (2001) Anxiety in high-functioning children with autism. Autism 5: 277-286.

9 Bellini S (2004) Social skills deficits and anxiety in high-functioning adolescents with autism spectrum disorders. Focus Autism Other Dev Disabl 19: 78-86.

10 Burnette $C P$, Mundy PC, Meyer JA, Sutton SK, Vaughan AE, et al. (2005) Weak central coherence and its relation to theory of mind and anxiety in autism. J Autism Dev Disord 35: 63-73. 
11 Green J, Gilchrist A, Burton D, Cox A (2000) Social and psychiatric functioning in adolescents with Asperger syndrome compared with conduct disorder. J Autism Dev Disord 30: 279-293.

12 Happe F, Briskman J, Frith U (2001) Exploring the cognitive phenotype of autism: Weak 'central coherence' in parents and siblings of children with autism: I. Experimental tests. J Child Psychol Psychiatry 42: 299-307.

13 Morgan B, Maybery M, Durkin K (2003) Weak central coherence, poor joint attention, and low verbal ability: Independent deficits in early autism. Dev Psychol 39: 646-656.

14 Ginsburg G, La Greca AM, Silverman WK (1998) Social anxiety in children with anxiety disorders: Relation with social and emotional functioning. J Abnorm Child Psychol 26: 175-185.

15 Velting O, Setzer J, Albano A (2004) Update on advances in assessment and cognitive-behavioural treatment of anxiety disorders in children and adolescents. Prof Psychol Res Pr 35: 42-54.

16 Kymissis P, Halperin D (1995) Group therapy with children and adolescents. Washington DC: American Psychiatric Press.

17 MacDonald E, Chowdhury U, Dabney J, Wolpert Miranda, Stein Samuel (2003) A social skills group for children. Emot Behav Diffic 8: 43-52.

18 Yalom ID, Leszcz M (2005) The Theory and Practice of Group Psychotherapy. $5^{\text {th }}$ edn. New York: Basic books.

19 Dennison S (2008) Measuring the treatment outcome of short-term school-based social skills groups. Soc Work Groups 31: 307-328.

20 Attwood T (1999) Modifications to cognitive behaviour therapy to accommodate the cognitive profile of people with Asperger's Syndrome. Autism Community Training (ACT).

21 Anderson S, Morris J (2006) Cognitive behaviour therapy for people with Asperger syndrome. Behav Cogn Psychother 34: 293-303.

22 Shindler S, Tangelder M (2010) Beneath the mask: A group therapy model supporting children infected with and affected by HIV/AIDS Soc Work Groups 33: 308-322.

23 Eichstedt, Wilde, Hols Tucker, Collins (2006) The worry warriors program. In: Garland EJ, Clark SL, Earle V (eds) Taming worry dragons: A manual for children, parents, and other coaches. $2^{\text {nd }}$ edn. Vancouver: BC Children's Hospital.

24 Juliana IT, Julie AE, Veitch WV, Elizabeth P, Sharon B, et al. (2011) Group cognitive-behavioural therapy for anxiety in a clinic setting: Does child involvement predict outcome? Behav Ther 42: 306-322.

25 Duncan AW, Klinger LG (2010) Autism spectrum disorders: Building social skills in group, school, and community settings. Soc Work Groups 33: 175-193.
26 Wood JW, Drahota A, Sze K, Har K, Chiu A, et al. (2009) Cognitive behavioural therapy for anxiety in children with autism spectrum disorders: A randomised, controlled trial. J Child Psychol Psychiatry 50: 224-234.

27 Mundy PC, Henderson HA, Inge AP, Coman DC (2007) The modifier model of autism and social development in higher functioning children. Res Pract Persons Severe Disabl 32: 124-139.

28 Liber JM, Van Widenfelt BM, Utens EM, Ferdinand RF, Van der Leeden AJ, et al. (2008) No differences between group versus individual treatment of childhood anxiety disorders in a randomised clinical trial. J Child Psychol Psychiatry 49: 886-893.

29 Chalfant A, Rapee R, Carroll L (2006) Treating anxiety disorders in children with high functioning autism spectrum disorders: $A$ controlled trial. J Autism Dev Disord 37: 1842-1857.

30 Sofronoff K, Attwood T, Hinton S (2005) A randomised controlled trial of CBT intervention for anxiety in children with Asperger syndrome. J Child Psychol Psychiatry 46: 1152-1160.

31 Reaven JA, Blakely-Smith A, Nichols S, Dasari M, Flanigan E, et al. (2009) Cognitive-behavioural group treatment for anxiety symptoms in children with high-functioning autism spectrum disorders: A pilot study. Focus Autism Other Dev Disabl 24: 27-37.

32 Reaven J, Blakeley-Smith A, Culhane-Shelburne K, Hepburn S (2012) Group cognitive behaviour therapy for children with high-functioning autism spectrum disorders and anxiety: A randomized trial. J Child Psychol Psychiatry 53: 410-419.

33 White SW, Ollendick T, Albano AM, Oswald D, Johnson C, et al. (2013) Randomized controlled trial: Multimodal anxiety and social skill intervention for adolescents with autism spectrum disorder. J Autism Dev Disord 43: 382-394.

34 Lord C, Rutter M, DiLavore P, Risi S (2000) Autism diagnostic observation schedule (ADOS) manual. Los Angeles, CA: Western Psychological Services.

35 Lord C, Rutter M, Le Couteur A (1994) Autism diagnostic interviewrevised: A revised version of the diagnostic Interview for caregivers of individuals with possible pervasive developmental disorders. J Autism Dev Disord 24: 659-685.

36 Silverman W, Albano A (1996) Anxiety Disorders Interview Schedule (ADIS-IV): Child and parent interview schedules. San Antonio, TX, Psychological Corporation.

37 Baker MJ, Koegel RL, Koegel LK (1998) Increasing the social behaviour of young children with autism using their obsessive behaviours. Res Pract Pers Sev Disabil 23: 300-308.

38 Scarpa A, White SW, Attwood T (2016) CBT for children and Adolescents with high-functioning autism spectrum disorders. The Guilford Press, p: 329. 